\title{
Metrical Coordinates in Non-Euclidean Geometry
}

\author{
By D. M. Y. Sommerville,
}

Victoria University College, Wellington, N.Z.

(Received 15th July, 1931. Read 5th February, 1932.)

$\S 1$. The coordinates considered are linear, i.e. in a plane the equation of a straight line, and in space the equation of a plane, is linear in the coordinates. We shall first consider point-coordinates in plane geometry, taking elliptic geometry as typical, with spaceconstant unity.

\$1.1. The general linear or projective coordinates are defined with respect to a fundamental triangle $X Y Z$ and a unit-point $U \equiv[1,1,1]$. Let $P \equiv[x, y, z]$ be any point; $L, M, N$ the feet of the perpendiculars from $P ; L_{0}, M_{0}, N_{0}$ those from $U$, on the sides of the triangle. Let $P U$ cut the sides of the triangle in $A, B, C$. Then ${ }^{1}$

$$
\begin{aligned}
\frac{x}{y} & =\text { the cross-ratio }(A B, P U) \\
& =\frac{\sin A P}{\sin B P} / \frac{\sin A U}{\sin B U} .
\end{aligned}
$$

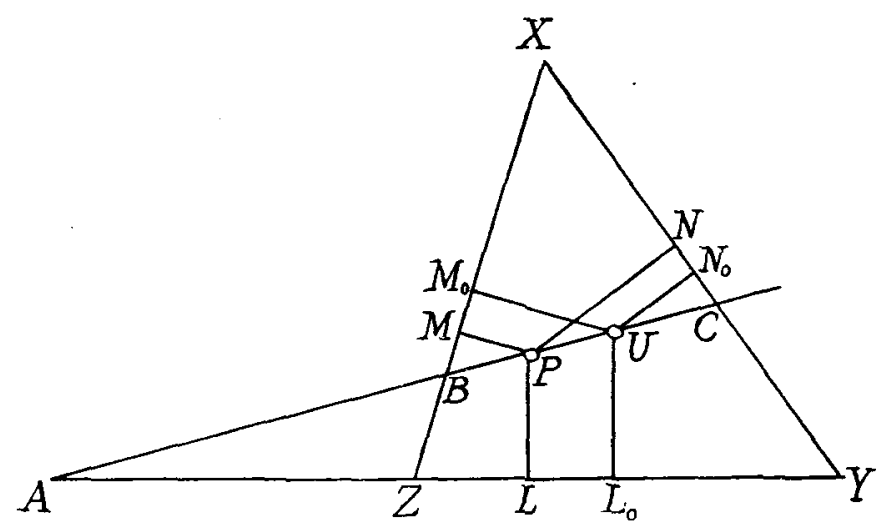

${ }^{1}$ See, for example, Castelnuovo: Lexioni di geometria analitica (6th ed. 1924), p. 229 ; or the author's Geometry of $n$ dimensions (Methuen, 1929), p. 55. 
Now $\sin L P=\sin A P \sin L A P$,

therefore $\sin L_{0} U=\sin A U \sin L_{0} A U$,

Similarly

$$
\frac{\sin A P}{\sin A U}=\frac{\sin L P}{\sin L_{0} U} .
$$

Hence

$$
\frac{\sin B P}{\sin B U}=\frac{\sin M P}{\sin M_{0} U} .
$$

and therefore

$$
\frac{x}{y}=\frac{\sin L P}{\sin L_{0} U} / \frac{\sin M P}{\sin M_{0} U},
$$

$$
\rho x=\frac{\sin L P}{\sin L_{0} U}, \quad \rho y=\frac{\sin M P}{\sin M_{0} U}, \quad \rho z=\frac{\sin N P}{\sin N_{0} U},
$$

where $\rho$ is a factor of proportionality.

$\$ 1.2$. If we assume next that the point-equation of the Absolute is a homogeneous quadratic in $x, y, z$ :

$$
(x x) \equiv a_{0} x^{2}+b_{0} y^{2}+c_{0} z^{2}+2 f_{0} y z+2 g_{0} z x+2 h_{0} x y=0,
$$

the distance $d$ between two points $(x),\left(x^{\prime}\right)$ is given by

$$
\cos d=\frac{\left(x x^{\prime}\right)}{\sqrt{ }(x x) \sqrt{ }\left(x^{\prime} x^{\prime}\right)} .
$$

Also if the tangential equation of the Absolute is

$$
(\xi \xi) \equiv A_{0} \xi^{2}+\ldots+2 F_{0} \eta \zeta+\ldots=0,
$$

the angle $\theta$ between two lines $(\xi),\left(\xi^{\prime}\right)$ is given by

$$
\cos \theta=\frac{\left(\xi \xi^{\prime}\right)}{\sqrt{ }(\xi \xi) \sqrt{ }\left(\xi^{\prime} \xi^{\prime}\right)} .
$$

Further, the distance between the point $(x)$ and the line $(\xi)$ is the complement of the distance between $(x)$ and the absolute pole of $(\xi)$, i.e. the point $\left[x^{\prime}, y^{\prime}, z^{\prime}\right] \equiv\left[A_{0} \xi+H_{0} \eta+G_{0} \zeta, \ldots\right]$.

Now

$$
\begin{aligned}
\left(x x^{\prime}\right) & =x^{\prime}\left(a_{0} x+h_{0} y+g_{0} z\right)+\ldots \\
& =\Delta(\xi x+\eta y+\zeta z),
\end{aligned}
$$

where $\Delta$ is the discriminant of $(x x)$. Also

$$
\left(x^{\prime} x^{\prime}\right)=\Delta\left\{\xi\left(A_{0} \xi+H_{0} \eta+G_{0} \zeta\right)+\ldots\right\}=\Delta(\xi \xi) .
$$

Hence we see that

$$
\sin p=\frac{\Delta^{\frac{1}{2}}(\xi x+\eta y+\zeta z)}{\sqrt{ }(x x) \sqrt{ }(\xi \xi)}
$$


$\S 1.3$. The distance from $[x, y, z]$ to the line $x=0$, i.e. the line $[1,0,0]$, is then given by

$$
\sin L P=\frac{\Delta^{\frac{1}{2}} x}{\sqrt{ }(x x) \cdot A_{0}^{\frac{1}{2}}} .
$$

Comparing this with (1.11), we have

$$
A_{0}=k / \sin ^{2} p_{0}
$$

where $p_{0}=L_{0} U$ and $k=\Delta / \rho^{2}(x x)$.

Again, if $a, b, c$ are the lengths of the sides of the triangle of reference, we have

Therefore

$$
\cos a=\frac{f_{0}}{\sqrt{ }\left(b_{0} c_{0}\right)} .
$$

$$
k / \sin ^{2} p_{0}=A_{0}=b_{0} c_{0}-f_{0}^{2}=b_{0} c_{0} \sin ^{2} a \text {. }
$$

Hence

$$
\begin{aligned}
& a_{0}=k^{\prime} \sin ^{2} a \sin ^{2} p_{0}, \\
& f_{0}=k^{\prime} \sin q_{0} \sin r_{0} \sin b \sin c \cos a,
\end{aligned}
$$

where $k^{\prime}=a_{0} b_{0} c_{0} / k$.

The point-equation of the Absolute is therefore $x^{2} \sin ^{2} p_{0} \sin ^{2} a+\ldots+2 y z \sin q_{0} \sin r_{0} \sin b \sin c \cos a+\ldots=0$ and the tangential equation is $\xi^{2} \sin ^{2} q_{0} \sin ^{2} r_{0}+\ldots+2 \eta \zeta \sin ^{2} p_{0} \sin q_{0} \sin r_{0}(\cos b \cos c-\cos a)+\ldots=0$ or $\quad \xi^{2} / \sin ^{2} p_{0}+\ldots-2 \cos A \eta \xi / \sin q_{0} \sin r_{0}-\ldots=0$. where $A, B, C$ are the angles of the triangle of reference.

\$1.4. Similarly if the line $(\xi)$ cuts the sides of the triangle of reference in $A, B, C$, and the unit-line cuts the sides in $A_{0}, B_{0}, C_{0}$, then

$$
\frac{\eta}{\zeta}=\left(Y Z, A A_{0}\right)=\frac{\sin Y A}{\sin Z A} / \frac{\sin Y A_{0}}{\sin Z A_{0}} .
$$

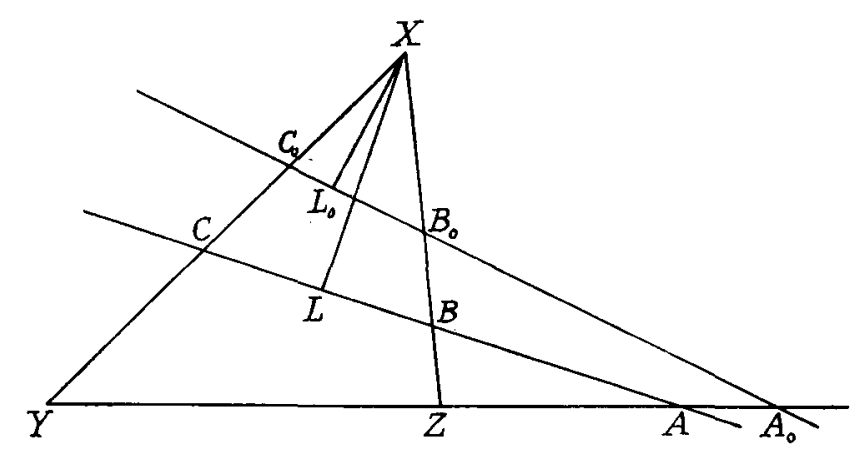


Let $L, M, N$ be the feet of the perpendiculars from $X, Y, Z$ on the line $(\xi)$, and $L_{0}, M_{0}, N_{0}$ those of the unit-line. Then we find

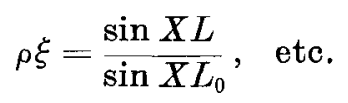

The unit-line is determined by the unit-point, being polar and pole with regard to the triangle of reference, and we find

$$
\rho^{\prime} \sin X L_{0}=\sin p_{0} \sin A, \text { etc. }
$$

Hence the general point-coordinates are certain multiples $l, m, n$ of the sines of the distances of the point from the sides of the triangle, and the related line-coordinates are multiples $l / \sin a, m / \sin b, n / \sin c$ of the sines of the distances of the line from the vertices.

\section{§1.5. The Analogue of Trilinears.}

We consider now the special systems of metrical coordinates which correspond to trilinears, areals, and Cartesians.

In trilinears the unit-point is the centre of the inscribed circle of the triangle of reference, so that $p_{0}=q_{0}=r_{0}$, and the point-equation of the Absolute is

$$
x^{2} \sin ^{2} a+\ldots+2 y z \sin b \sin c \cos a+\ldots=0
$$

where $a, b, c$ are the sides of the triangle of reference. The coordinates $x, y, z$ are

$$
\rho x=\sin L P, \quad \rho y=\sin M P, \quad \rho z=\sin N P .
$$

The line-equation of the Absolute is

$$
\xi^{2}+\eta^{2}+\zeta^{2}-2 \eta \zeta \cos A-2 \zeta \xi \cos B-2 \xi \eta \cos C=0 .
$$

\$1.6. The Analogue of Areals.

In areals the point-coordinates are

$$
\rho x=\sin L P \sin a, \quad \rho y=\sin M P \sin b, \quad \rho z=\sin N P \sin c .
$$

The point-equation of the Absolute is

$$
x^{2}+y^{2}+z^{2}+2 y z \cos a+2 z x \cos b+2 x y \cos c=0
$$

and the line-equation is

$$
\xi^{2} \sin ^{2} A+\ldots-2 \eta \zeta \sin B \sin C \cos A-\ldots=0 .
$$

If $X U, Y U, Z U$ cut the opposite sides of the triangle in $D, E, F$, it is readily found that $\cos Y D=\cos \frac{1}{2} a=\cos D Z$. Hence $D, E, F$ are the mid-points of the sides, and $U$ is the centroid of the triangle. 


\section{\$1.7. Cartesian coordinates.}

For the analogue of Cartesian coordinates we modify the areal system by choosing the side $X Y$ as the absolute pole of the opposite vertex $Z$. Then $b=c=\frac{1}{2} \pi=B=C$, and $a=A$. We shall change the notation, calling $Z$ the origin $O$, and let $A$, the angle between the axes, be denoted by $\omega$.

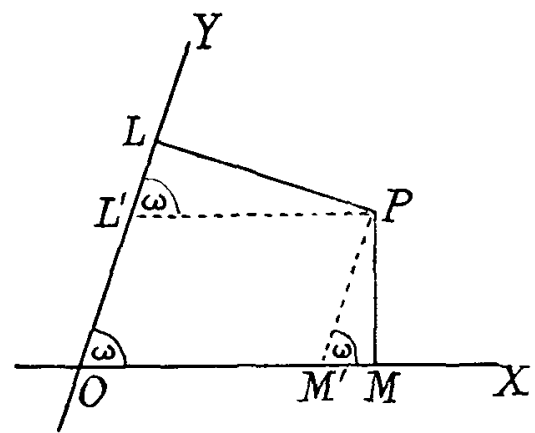

Then

$$
\rho^{\prime} x=\sin L P, \quad \rho^{\prime} y=\sin M P, \quad \rho^{\prime} z=\cos O P \sin \omega
$$

or, putting $\rho^{\prime}=\rho \sin \omega$,

$$
\rho x=\sin L P / \sin \omega, \quad \rho y=\sin M P / \sin \omega, \quad \rho z=\cos O P .
$$

Now if $L^{\prime} P$ and $M^{\prime} P$ are drawn so that angle $P L^{\prime} L=\omega=P M^{\prime} M$, then

$$
\rho x=\sin L^{\prime} P, \quad \rho y=\sin M^{\prime} P, \quad \rho z=\cos O P .
$$

The point-equation of the Absolute is

and the line-equation

$$
x^{2}+y^{2}+z^{2}+2 x y \cos \omega=0
$$

$$
\xi^{2}+\eta^{2}+\zeta^{2} \sin ^{2} \omega-2 \xi \eta \cos \omega=0
$$

When $\omega=\frac{1}{2} \pi$ we have the coordinates of Weierstrass, the analogue of rectangular cartesians.

§2. Metrical coordinates in three dimensions. The extension to three dimensions is perhaps not quite obvious owing to the variety of angular magnitudes: edges of the tetrahedron, dihedral angles, and face-angles.

\$2.1. As in plane geometry, if $L, M, N$ are the feet of the perpendiculars from $P$ on the faces of the tetrahedron of reference, $L_{0}, M_{0}, N_{0}$ those for the unit-point $U$, we have

$$
\rho x=\sin L P / \sin L_{0} U=\sin L P / \sin p_{0}, \text { etc. }
$$


Let the point-equation of the Absolute be

$$
\begin{gathered}
(x x) \equiv a_{0} x^{2}+b_{0} y^{2}+c_{0} z^{2}+d_{0} w^{2}+2 f_{0} y z+2 g_{0} z x+2 h_{0} x y \\
+2 l_{0} x w+2 m_{0} y w+2 n_{0} z w=0 .
\end{gathered}
$$

Let the edges of the tetrahedron be $X Y=a_{12}$, etc., the dihedral angle between the faces $x=0$ and $y=0$, i.e. at the edge $Z W$, be $a_{12}$, etc.

The tangential equation of the Absolute is

$$
(\xi \xi)=A_{0} \xi^{2}+\ldots+2 F_{0} \eta \zeta+\ldots+2 L_{0} \xi \omega+\ldots=0,
$$

where, as usual, capital letters denote the cofactors of the corresponding small letters in the determinant $\Delta$.

Then, as before, we have

and also

$$
A_{0}=k / \sin ^{2} p_{0}, \text { etc. }
$$

Hence

$$
\cos a_{12}=h_{0} / \sqrt{ }\left(a_{0} b_{0}\right) \text {, etc. }
$$

$$
\begin{aligned}
\frac{k}{\sin ^{2} p_{0}}=A_{0} & =\left|\begin{array}{lll}
b_{0} & f_{0} & m_{0} \\
f_{0} & c_{0} & n_{0} \\
m_{0} & n_{0} & d_{0}
\end{array}\right|=b_{0} c_{0} d_{0}\left|\begin{array}{lll}
1 & \cos a_{23} & \cos a_{24} \\
\cos a_{23} & 1 & \cos a_{34} \\
\cos a_{24} & \cos a_{34} & 1
\end{array}\right| \\
& =b_{0} c_{0} d_{0} S_{1}^{2}, \quad \text { say. }
\end{aligned}
$$

Then

$$
\begin{aligned}
& a_{0}=k^{\prime} \sin ^{2} p_{0} S_{1}^{2}, \ldots \\
& f_{0}=k^{\prime} \cos a_{23} \sin q_{0} \sin r_{0} S_{2} S_{3}, \ldots \\
& l_{0}=k^{\prime} \cos a_{14} \sin p_{0} \sin s_{0} S_{1} S_{4}, \ldots
\end{aligned}
$$

Hence the point-equation of the Absolute is

$$
\begin{gathered}
x^{2} \sin ^{2} p_{0} S_{1}^{2}+\ldots+2 y z \sin q_{0} \sin r_{0} S_{2} S_{3} \cos a_{23}+\ldots \\
+2 x w \sin p_{0} \sin s_{0} S_{1} S_{4} \cos a_{14}+\ldots \ldots=0 .
\end{gathered}
$$

For the tangential equation we have already found $A_{0}, \ldots, D_{0}$. To find $F_{0}$ we have

$$
-\cos \alpha_{23}=F_{0} / \sqrt{ }\left(B_{0} C_{0}\right) \text {. }
$$

Hence the tangential equation is

$$
\begin{aligned}
\xi^{2} / \sin ^{2} p_{0}+\ldots- & 2 \eta \zeta \cos \alpha_{23} / \sin q_{0} \sin r_{0}-. . \\
& -2 \xi \omega \cos \alpha_{14} / \sin p_{0} \sin s_{0}-\ldots=0 .
\end{aligned}
$$

\section{§2.2. Trilinears.}

Taking $U$ as the centre of the inscribed sphere, we have $p_{0}=q_{0}=r_{0}=s_{0}$, and

$$
\rho x=\sin L P, \text { etc. }
$$


The point-equation of the Absolute is

$$
x^{2} S_{1}^{2}+\ldots 2 y z S_{2} S_{3} \cos a_{23}+\ldots+2 x w S_{1} S_{4} \cos a_{14}+\ldots=0
$$

and the tangential equation is

$$
\xi^{2}+\ldots-2 \eta \zeta \cos \alpha_{23}-\ldots-2 \xi \omega \cos \alpha_{14}-\ldots=0
$$

§2.3. Areals.

Choosing $U$ so that

$$
\begin{gathered}
S_{1} \sin p_{0}=S_{2} \sin q_{0}=S_{3} \sin r_{0}=S_{4} \sin s_{0}, \\
\rho x=S_{1} \sin L P, \text { etc., }
\end{gathered}
$$

then the point-equation of the Absolute is

$$
x^{2}+\ldots+2 y z \cos a_{23}+\ldots+2 x w \cos a_{14}+\ldots=0
$$

and the tangential equation

$\xi^{2} S_{1}^{2}+\ldots-2 \eta \zeta S_{2} S_{3} \cos \alpha_{23}-\ldots-2 \xi \omega S_{1} S_{4} \cos \alpha_{14}-\ldots=0$.

We find that the plane $U X Y$ bisects the edge $Z W$, etc., so that $U$ is the centroid of the tetrahedron.

\section{§2.4. Cartesians.}

Modifying the "areal" system by choosing $X Y Z$ as the absolute polar of $W$ (or the origin $O$ ), we have $a_{14}=a_{24}=a_{34}=\frac{1}{2} \pi=\alpha_{14}=\alpha_{24}=\alpha_{34}$; write also for the plane-angles between the axes $O X, O Y, O Z$, $a_{23}=\lambda, a_{31}=\mu, a_{12}=\nu$.

The functions $S_{1}^{2}, S_{2}^{2}, S_{3}^{2} \operatorname{simplify}$ to $\sin ^{2} \lambda, \sin ^{2} \mu, \sin ^{2} \nu$ : hence

$$
\begin{gathered}
\rho x=\sin L P \sin \lambda, \quad \rho y=\sin M P \sin \mu, \quad \rho z=\sin N P \sin \nu, \\
\rho w=\cos O P . S_{4} .
\end{gathered}
$$

The point-equation of the Absolute becomes

$$
x^{2}+y^{2}+z^{2}+w^{2}+2 y z \cos \lambda+2 z x \cos \mu+2 x y \cos \nu=0,
$$

and the tangential equation

$$
\begin{gathered}
\xi^{2} \sin ^{2} \lambda+\eta^{2} \sin ^{2} \mu+\zeta^{2} \sin ^{2} \nu+\omega^{2} S_{4}^{2}-2 \eta \zeta \sin \mu \sin \nu \cos \alpha_{23} \\
-2 \zeta \xi \sin \nu \sin \lambda \cos \alpha_{31}-2 \xi \eta \sin \lambda \sin \mu \cos \alpha_{1: 2}=0
\end{gathered}
$$

Let $L, M, N$ be the angles which each coordinate-axis makes with the opposite coordinate-plane. Draw a sphere with centre $O$. The coordinate planes cut this in a spherical triangle $X^{\prime} Y^{\prime} Z^{\prime}$ whose sides are $\lambda, \mu, \nu$, angles, $\alpha_{23}, \alpha_{31}, \alpha_{12}$, and altitudes, $L, M, N$.

Now we have

$$
\sin L=\sin \mu \sin \alpha_{12},
$$

and

$$
\cos \nu=\cos \lambda \cos \mu+\sin \lambda \sin \mu \cos \alpha_{12} \text {. }
$$


Therefore, eliminating $\alpha_{12}$, we obtain

so that

$$
\sin ^{2} L=\sin ^{2} \mu\left\{1-\frac{(\cos \nu-\cos \lambda \cos \mu)^{2}}{\sin ^{2} \lambda \sin ^{2} \mu}\right\}
$$

$$
\begin{aligned}
\sin ^{2} L \sin ^{2} \lambda & =\sin ^{2} \lambda \sin ^{2} \mu-(\cos \nu-\cos \lambda \cos \mu)^{2} \\
& =1-\cos ^{2} \lambda-\cos ^{2} \mu-\cos ^{2} \nu+2 \cos \lambda \cos \mu \cos \nu \\
& =S_{4}^{2} .
\end{aligned}
$$

Hence $\quad \sin L \sin \lambda=\sin M \sin \mu=\sin N \sin \nu=S_{4}$.

The point-coordinates are

$$
\rho^{\prime} x=\sin L P \sin \lambda, \ldots, \rho^{\prime} w=S_{4} \cos O P,
$$

or, using (2.44),

$$
\rho x=\sin L P / \sin L, \ldots, \rho w=\cos O P .
$$

\$2.5. If $\alpha, \beta, \gamma$ are the angles which $O P$ makes with the coordinate-planes, then

Therefore

$$
\begin{aligned}
& \sin L P=\sin O P \sin a, \text { etc. } \\
& \cos O P=w / \sqrt{ }(x x) .
\end{aligned}
$$

$$
\begin{gathered}
w^{2}=\left(x^{2}+y^{2}+z^{2}+w^{2}+2 y z \cos \lambda+2 z x \cos \mu+2 x y \cos \nu\right) \cos ^{2} O P, \\
\rho^{\prime 2} w^{2} \sin ^{2} O P=\sin ^{2} O P\left(\sin ^{2} a \sin ^{2} \lambda+\sin ^{2} \beta \sin ^{2} \mu+\sin ^{2} \gamma \sin ^{2} \nu\right. \\
\quad+2 \sin \beta \sin \gamma \sin \mu \sin \nu \cos \lambda+\ldots) \cos ^{2} O P .
\end{gathered}
$$

Hence the direction-angles $\alpha, \beta, \gamma$ are connected by the identity

$$
\sin ^{2} \alpha \sin ^{2} \lambda+\ldots+2 \sin \beta \sin \gamma \sin \mu \sin \nu \cos \lambda+\ldots=S_{4}^{2} \text {. }
$$

When the coordinates are rectangular, $\lambda=\mu=\nu=\frac{1}{2} \pi$ and $S_{4}=1$; then

$$
\sin ^{2} \alpha+\sin ^{2} \beta+\sin ^{2} \gamma=1 .
$$

This indicates that, contrary to the usual custom, the directionangles should be defined as the angles which the radius-vector makes with the coordinate-planes, instead of with the coordinate-axes. The "direction-sines" $\sin a, \ldots$, are of course a system of "trilinear" coordinates in the spherical geometry about the point $O$.

§3.1. Extension to $n$ dimersions. In the extension to $n$ dimensions we shall alter the notation. Let the simplex of reference be $X_{0}, X_{1}, \ldots, X_{n}$; let the coordinates be $x_{0}, \ldots, x_{n}$; let the perpendicular from unit-point on to the coordinate-plane $x_{r}=0$ be $p_{r}$, and that from $P$ be $L_{r} P$. 
Then

$$
\rho x_{r}=\frac{\sin L_{r} P}{\sin p_{r}} .
$$

Let the lengths of the edges of the simplex be $X_{r} X_{s}=k_{r s}$, and the dihedral angle between the primes $x_{r}=0$ and $x_{s}=0$ be $\kappa_{r s}$.

Let the point-equation and tangential-equation of the Absolute be

Then

$$
\begin{aligned}
& (x x) \equiv \Sigma \Sigma a_{r s} x_{r} x_{s}=0, \\
& (\xi \xi) \equiv \Sigma \Sigma A_{r s} \xi_{r} \xi_{s}=0 .
\end{aligned}
$$

Hence

$$
\sin L_{r} P=\frac{\Delta^{\frac{1}{3}} x_{r}}{A_{r r}^{\frac{1}{2}} \sqrt{ }(x x)} .
$$

Also

$$
A_{r r}=k / \sin ^{2} p_{r}
$$

$$
\cos k_{r s}=\frac{a_{r s}}{\sqrt{ }\left(a_{r r} a_{s s}\right)}
$$

Expressing $A_{r r}$ as the cofactor of $a_{r r}$ in $\Delta$, we have

where

$$
A_{r r} a_{r r}=a_{00} a_{11} \ldots a_{n n} S_{n, r}^{2},
$$

Then

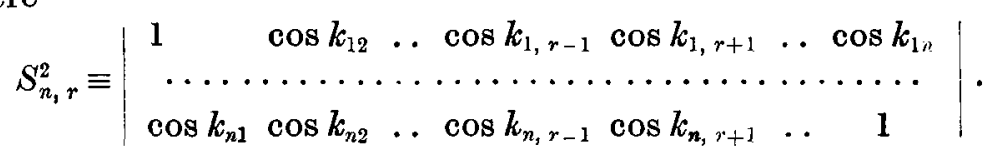

$$
\left.\begin{array}{l}
a_{r r}=k^{\prime} \sin ^{2} p_{r} S_{n, r}^{2} \\
a_{r s}=k^{\prime} \sin p_{r} \sin p_{s} S_{n, r} S_{n, s} \cos k_{r s}
\end{array}\right\}
$$

\$3.2. If $U$ is the centroid of the simplex (areals)

and

$$
\begin{aligned}
& \sin p_{r} \cdot S_{n, r}=\text { const. } \\
& \rho x_{r}=S_{n, r} \sin L_{r} P
\end{aligned}
$$

and the point-equation of the Absolute becomes

$$
\Sigma x_{r}^{2}+2 \Sigma x_{r} x_{s} \cos k_{r s}=0 .
$$

The tangential equation is

$$
\sum S_{n, r}^{2} \xi_{r}^{2}-2 \Sigma \xi_{r} \xi_{s} S_{n, r} S_{n, s} \cos \kappa_{r s}=0
$$

§3.3. To obtain the Cartesian system take the prime $x_{0}=0$ as the absolute polar of $X_{0}$ (or $O$ ), so that $k_{0 r}=\frac{1}{2} \pi=\kappa_{0 r}$, where $k_{r s}$ is the angle between the lines $O X_{r}$ and $O X_{s}$. The point-equation of the Absolute is then

$$
x_{0}^{2}+\sum x_{r}^{2}+2 \Sigma x_{r} x_{s} \cos k_{r s}=0 \quad(r \neq s=1,2, \ldots, n) .
$$


For the tangential equation the coefficient of $\xi_{0}^{2}$ is $S_{\mu, 0}^{2}$. That of $\xi_{1}^{2}$ is

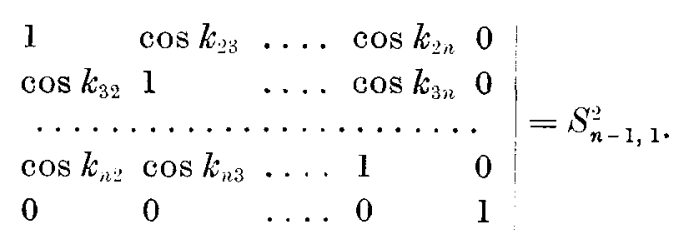

The tangential equation is then

$$
S_{n, 0}^{2} \xi_{0}^{2}+\Sigma S_{n-1, r}^{2} \xi_{r}^{2}-2 \Sigma \xi_{r} \xi_{s} S_{n-1, r} S_{n-1, s} \cos \kappa_{r s}=0
$$

The point-coordinates are

$$
\left.\begin{array}{l}
\rho x_{0}=\frac{\sin L_{0} P}{\sin p_{10}}=S_{n, 0} \cos O P, \\
\rho x_{r}=S_{n-1, r} \sin L_{r} P .
\end{array}\right\}
$$

Let $\theta_{r}$ be the angle which $O X_{r}$ makes with the opposite coordinate-prime. Draw a hypersphere round $O$ and we obtain a spherical simplex of $n-1$ dimensions whose edges are the angles $k_{r s}$ and altitudes $\theta_{i .}$. Applying to this the formula for distance in $n-1$ dimensions we have

and $\Delta_{n-1}=S_{n, 0}^{2}$.

$$
\sin \theta_{r}=\frac{\Delta_{n-1}^{\frac{1}{2}}}{S_{p-1, r}}
$$

Hence dividing the coordinates by $S_{n, 0}$, we have

$$
\left.\begin{array}{l}
\rho x_{i j}=\cos O P, \\
\rho x_{r}=\frac{S_{n-1, r} \sin L_{r} P}{S_{i, 0}}=\sin L_{r} P \sin \theta_{r}
\end{array}\right\} .
$$

